\title{
THE CALCULATION OF HEAT FLOW IN MELTING SOLIDS
}

\author{
BY \\ MARK LOTKIN \\ AVCO Research and Development, Wilmington, Mass.
}

I. Introduction. The numerical integration of certain heat conduction equations was treated by the author in [1]. The case considered there dealt with the transfer of heat in a one-dimensional finite slab composed of materials possessing variable thermal properties, subject to appropriate initial and boundary conditions.

The purpose of this note is to extend the previous discussion to include the phenomenon of melting. In carrying out this extension it turns out to be advisable to modify the numerical technique of integration used in [1] by going to unequal subdivisions in both space and time variables. Near the melting surface, where the changes of temperature may be very pronounced, it is advantageous to choose rather small integration steps. Farther away from the melting surface, on the other hand, where the temperature may change rather slowly, a more economic treatment of the integration process may be achieved by taking relatively large intervals in the independent variables.

In the following sections, then, the physical situation is first defined, and the governing mathematical equations are stated. These differential equations are next replaced by analogous difference equations, and the resulting truncation terms are noted. It turns out that the stability characteristics of the method are quite similar to those established in [1].

As numerous calculations carried out on the IBM 704 machine by means of a program based on some of the equations discussed here have shown, the procedure derived in this note leads to quite satisfactory results.

II. System of differential equations. Let us consider, then, the one-dimensional conduction of heat in a solid slab consisting of one material, and extending from $x=0$ to $x=a$, with the surface at $x=a$ to be insulated. Through the face initially located at $x=0$ let heat enter at the rate $q(t)$. Let it further be assumed that the amount of heat flowing through the frontal face is at times sufficiently large to raise the frontal temperature to the melting temperature $u_{m}$ of the material, thus actually setting off the melting process, and continuing it until the temperature drops temporarily below $u_{m}$. If, upon melting, the liquid material is assumed to be removed instantaneously the "frontal" face of the solid is seen to move forward, to a position $x=s(t)$ at time $t$. Of interest then is the temperature $u=u(x, t)$, and the amount $s(t)$ melted.

The basic equations governing the melting problem described above are:

$$
c \rho \frac{\partial u}{\partial t}=\frac{\partial}{\partial x}\left(k \frac{\partial u}{\partial x}\right) \quad s(t) \leq x \leq a, \quad t>0,
$$

where $c, \rho, k$ denote known functions of the temperature $u$.

Initially,

$$
u(x, 0)=f(x) \leq u_{m} \quad 0 \leq x \leq a, \quad t=0 .
$$

Received February 10, 1959; revised manuscript received July 10, 1959. 
At the frontal face

with

$$
\left.\begin{array}{l}
-k(\partial u / \partial x)=q(t)-\rho F s^{\cdot} \\
s^{\cdot}(t)=0 \text { for } u(s, t)<u_{m} \\
s(t) \geq 0 \text { for } u(s, t)=u_{m}
\end{array}\right\} x=s(t), \quad t>0 .
$$

At the back face,

$$
(\partial u / \partial x)=0, \quad x=a, \quad t>0 .
$$

As pointed out by Landau [2], the numerical treatment of the problem is simplified somewhat by the removal of the moving boundary, by means of the transformation

$$
\xi=(x-s) /(a-s) \text {. }
$$

Clearly above equations then become.

$$
\begin{gathered}
u(x, t)=U(\xi, t), \\
c \rho\left[\frac{\partial U}{\partial t}+\frac{\xi-1}{a-s} s^{\cdot} \frac{\partial U}{\partial \xi}\right]=\frac{1}{(a-s)^{2}} \frac{\partial}{\partial \xi}\left(k \frac{\partial U}{\partial \xi}\right), \quad 0 \leq \xi \leq 1, \quad t>0, \\
U(\xi, 0)=f(\xi) \leq u_{m}, \quad 0 \leq \xi \leq 1, \quad t=0,
\end{gathered}
$$

with

$$
\begin{aligned}
& -\frac{k}{a-s}\left(\frac{\partial U}{\partial \xi}\right)=q(t)-\rho F s \\
& \left.s^{\prime}(t)=0 \text { for } U(0, t)<u_{m}\right\}=0, \quad t>0 \\
& s^{\prime}(t) \geq 0 \text { for } U(0, t)=u_{m} \\
& (\partial U / \partial \xi)=0 \quad \xi=1, \quad t>0 .
\end{aligned}
$$

III. Definitions and assumptions. Let us assume that $n+1$ values $\xi_{i}, i=0,1, \cdots n$ are given in $0 \leq \xi \leq 1$, with $\xi_{0}=0, \xi_{n}=1$. Further, let $\nabla_{i} \xi \equiv \nabla_{i}=\xi_{i}-\xi_{i-1}, \nabla_{i} t=$ $t_{i}-t_{i-1}$ for $i=1,2, \cdots n, j=1,2, \cdots ; t_{0}=0$, and $U\left(\xi_{i}, t_{j}\right)=U_{i j}, c\left(U_{i j}\right)=$ $c_{i j}, q\left(t_{i}\right)=q_{i}, \alpha=k(c \rho)^{-1}$, and $r_{i},=\alpha_{i i} \nabla_{i+1} t\left[\left(a-s_{i}\right) \nabla_{i+1} \xi\right]^{-2}$, with $\nabla_{n+1} \xi=$ $\nabla_{n} \xi$ for $i=n$.

In the subsequent numerical treatment extensive use is made of the relationships:

$$
\frac{1}{m !}\left(\frac{d^{m} w}{d z^{m}}\right)_{0}=\sum_{i=0}^{n-m} \sigma_{i}^{(m)}\left(\frac{D^{(i+m)} w}{D z^{i+m}}\right)_{i+m, 0}+\text { Const }\left(\frac{d^{n+1} w}{d z^{n+1}}\right)|\tau|^{n+1-m}
$$

which expresses the presumably existing derivatives of an arbitrary differentiable function $w(z)$ evaluated at $z=z_{0}$ in terms of the divided differences based on the points $\left(z_{i}, w_{i}\right), i=0,1,2, \cdots$ :

$$
\begin{gathered}
(D w / D z)_{10} \equiv\left(w_{1}-w_{0}\right) /\left(z_{1}-z_{0}\right) \\
\left(D^{2} w / D z^{2}\right)_{20} \equiv\left[(D w / D z)_{21}-(D w / D z)_{10}\right] /\left(z_{2}-z_{0}\right), \quad \text { etc.; }
\end{gathered}
$$

in Eq. (10) $\sigma_{i}^{(m)}$ denotes the $i$ th symmetric function of $\tau_{0}, \tau_{1}, \cdots, \tau_{i+m-1} ; \tau_{\mathbf{p}} \equiv z-z_{\boldsymbol{p}}$, $\tau=\max \left|z-z_{p}\right|$, and $\left(d^{n+1} w / d z^{n+1}\right)$ is to be evaluated at an intermediate value $\zeta$.

*The symbol $s^{*}$ denotes $d s / d t$. 
Thus,

$$
\begin{gathered}
\left(\frac{\partial U}{\partial \xi}\right)_{i, i+1}=\frac{U_{i+1, i+1}-U_{i, j+1}}{\nabla_{i+1}}-\nabla_{i+1}\left(\frac{D^{2} U}{D \xi^{2}}\right)_{i+2, i} \\
+\nabla_{i+1}\left(\nabla_{i+1}+\nabla_{i+2}\right)\left(\frac{D^{3} U}{D \xi^{3}}\right)_{i+3, i}+C_{1}|\nabla \xi|^{3} \\
\left(\frac{\partial U}{\partial t}\right)_{i, i+1}=\frac{U_{i, j+1}-U_{i i}}{\nabla_{i+1} t}-\nabla_{i+1} t\left(\frac{D^{2} U}{D t^{2}}\right)_{i+2, j}+C_{2}(\nabla t)^{2} \\
\frac{1}{2}\left(\frac{\partial^{2} U}{\partial \xi^{2}}\right)_{i, i+1}=\left(\frac{D^{2} U}{D \xi^{2}}\right)_{i+2, i}+C_{3}|\nabla \xi|
\end{gathered}
$$

with

$$
|\nabla \xi|=\max _{p=0,1, \cdots, n}\left|\nabla_{p} \xi\right|, \quad \nabla t=\max _{i=0,1,2, \cdots, j} \nabla_{i} t .
$$

Alternately, clearly also

$$
\begin{gathered}
-\left(\frac{\partial U}{\partial \xi}\right)_{i, i+1}=\frac{U_{i-1, j+1}-U_{i, j+1}}{\nabla_{i}}-\nabla_{i}\left(\frac{D^{2} U}{D \xi^{2}}\right)_{i-2, i}+C_{1}^{\prime}(\nabla \xi)^{2} \\
\frac{1}{2}\left(\frac{\partial^{2} U}{\partial \xi^{2}}\right)_{i, i+1}=\left(\frac{D^{2} U}{D \xi^{2}}\right)_{i+1, i-1}+C_{3}^{\prime}|\nabla \xi| .
\end{gathered}
$$

As to the nature of the function $x=s(t)$, it must clearly be continuous, and possess a non-negative derivative $\dot{s}(t)$ except for a finite number of values $t_{j}$ at which either $\dot{s}\left(t_{i+}\right)$ or $\dot{s}\left(t_{i-}\right)$ vanishes.

IV. Equation for the melting rate. For the following discussion it is convenient again to re-write Eq. [6] in the form:

$$
\frac{\partial^{2} U}{\partial \xi^{2}}=\frac{(a-s)^{2}}{\alpha}\left[\frac{\partial U}{\partial t}-\frac{1-\xi}{a-s} s \cdot \frac{\partial U}{\partial \xi}\right]-\frac{k^{\prime}}{k}\left(\frac{\partial U}{\partial \xi}\right)^{2},
$$

with $k^{\prime} \equiv d k / d U$.

In deriving suitable difference equations let us consider first the equation (8) governing the heated surface $\xi=0$. At time $t_{j+1}$, by Eq. (8a),

$$
-\frac{k_{0, j+1}}{a-s_{j+1}}\left(\frac{\partial U}{\partial \xi}\right)_{0, j+1}=q_{i+1}-\rho_{0, j+1} F s_{j+1}^{\cdot} \text {. }
$$

For the following considerations it is necessary to distinguish two cases, depending upon whether the surface temperature at $t_{i+1}$ is below or at the melting point $u_{m}$.

(i) $U_{0, i+1}<u_{m}$.

Then $\dot{s}_{i+1}=0, s_{i+1}=s_{i}$. Eq. (8a) becomes

$$
-(\partial U / \partial \xi)_{0, i+1}=\left(a-s_{j}\right) k_{0 i}^{-1} q_{i+1}+(a-s)\left(k^{-1}\right)_{\imath} q \nabla t .
$$

Now replacing $\left(U_{\xi}\right)_{0, i+1}$ by Eq. (12), and $\left(D^{2} U / D \xi^{2}\right)_{2,0}$ by Eq. (14), $\left(U_{\xi \xi}\right)_{0, i+1}$ by Eq. (17), $\left(U_{t}\right)_{0, i+1}$ by Eq. (13), there is obtained

$$
\left(1+2 r_{0 i}\right) U_{0, i+1}-2 r_{0 i} U_{1, i+1}=U_{0 i}+2 r_{0 i}\left(a-s_{i}\right) \nabla_{1} k_{0 i}^{-1} q_{i+1}+R_{1},
$$


with

$$
R_{1}=\alpha \nabla t \frac{k^{\prime}}{k}\left[\frac{U_{\xi}^{2}}{(a-s)^{2}}-2 \frac{U_{t} q \nabla t}{k(a-s) \nabla_{1}}\right]-\frac{\alpha \nabla_{1} \nabla t}{3(a-s)^{2}} U_{\xi \xi \xi}+\frac{\alpha_{t}}{\alpha} U_{t}(\nabla t)^{2} .
$$

(ii) $U_{0, j+1}=u_{m}$.

Now $\left(U_{t}\right)_{0, j+1}=0$. Eq. (8a) may be written as

$$
F \rho_{0 i} s_{i+1}=q_{i+1}+\frac{k_{0 i}}{a-s_{i+1}}\left(U_{\xi}\right)_{0, i+1}+\nabla t\left[\frac{k^{\prime} U_{t}}{a-s} U_{\xi}-F s^{\cdot} \rho_{t}\right] .
$$

Again replacing $\left(U_{\xi}\right)_{0, i+1}$ as indicated above under $(i)$, there results, after some calculations,

$$
F^{\prime} \rho_{0 j} s_{j+1}^{\cdot}=q_{i+1}-\frac{k}{\nabla_{1}\left(a-s_{i+1}\right)}\left(u_{m}-U_{1, i+1}\right)+\frac{k \nabla_{1}}{2 \alpha_{0, i}} s_{i+1}^{\cdot}\left(U_{\xi}\right)_{0, i+1}+R_{2}^{\prime},
$$

where

$R_{2}^{\prime}=\nabla_{1}\left[\frac{k^{\prime} U_{\xi}^{2}}{2(a-s)}+\frac{\text { const } k}{a-s} \nabla_{1} U_{\xi \xi \xi}\right]+\nabla t\left[\frac{k^{\prime} U_{t}}{a-s} U_{\xi}-F s^{\cdot} \rho_{t}\right]-\frac{\nabla_{1} \nabla t k s^{*}}{2} \frac{\alpha_{t}}{\alpha^{2}} U_{\xi}$.

Since $s_{i+1}=s_{i}+\nabla_{i+1} t s_{i+1}+0\left((\nabla t)^{2}\right)$, Eq. (19) may be replaced by

$$
s_{i+1}^{\cdot}=\frac{1}{F \rho_{0 i}}\left[q_{i+1}-\frac{k_{0 i}}{\nabla_{1}\left(a-s_{i}\right)}\left(u_{m}-U_{1, i+1}\right)+\frac{k_{0 j} \nabla_{1}}{2 \alpha_{0 i}} s_{i+1}\left(U_{\xi}\right)_{0, i+1}\right]+R_{2}^{\prime \prime},
$$

with

$$
F \rho R_{2}^{\prime \prime}=R_{2}^{\prime}+\frac{k s^{\cdot} \nabla t}{(a-s)^{2}}\left[U_{\xi}+\frac{\nabla_{1}}{2} U_{\xi \xi}\right] .
$$

In Eqs. (19) and (20) $(U \xi)_{0, i+1}$ is to be evaluated by means of Eq. (12). Since the accurate determination of $s^{\circ}$ is essential for the closeness of approximation of the solution of the difference equations to the solution of the differential equations, the evaluation of $(U \xi)_{0, j+1}$ by at least a four point formula seems advisable.

Equation (19) may be expressed in the form

$$
s_{i+1}^{\cdot}=A_{i+1}-B_{i+1}\left(a-s_{j+1}\right)^{-1}+R_{2},
$$

where

$$
\begin{aligned}
A_{i+1}= & q_{i+1}\left[F \rho_{0 i}-\frac{k_{0 i} \nabla_{1}\left(U_{\xi}\right)_{0, j+1}}{2 \alpha_{0 i}}\right]^{-1}>0, \\
B_{i+1}= & \frac{k_{0 i}\left(u_{m}-U_{1, i+1}\right)}{\nabla_{1}}\left[F \rho-\frac{k \nabla_{1}\left(U_{\xi}\right)}{2 \alpha}\right]^{-1}>0, \\
& R_{2}=R_{2}^{\prime}\left[F \rho-\frac{k \nabla_{1} U_{\xi}}{2 \alpha}\right]^{-1} .
\end{aligned}
$$

It is assumed here that $(U \xi)_{0, j+1} \leq 0$.

Similarly, from Eq. (20)

$$
s_{i+1}^{\cdot}=A_{i+1}-B_{i+1}\left(a-s_{i}\right)+R_{2}^{*} .
$$


The numerical solution of Eq. (21) may be accomplished by the iterative technique $\dagger$

$$
\begin{gathered}
\Sigma^{(n)}=A-B\left(a-\sigma^{(n)}\right)^{-1} \\
\sigma^{(n+1)}=\sigma_{i}+\left(\nabla_{i+1} t / 2\right)\left(\Sigma_{i}+\Sigma^{(n)}\right),
\end{gathered}
$$

for $n=0,1,2, \cdots$, with $\sigma^{(0)}=\sigma_{i}$ and $\sum \equiv d \sigma / d t$. It is seen that

$$
\sigma^{(n+1)}-\sigma^{(n)}=-\frac{B \nabla_{i+1} t}{2\left(a-\sigma^{(n)}\right)\left(a-\sigma^{(n-1)}\right)}\left(\sigma^{(n)}-\sigma^{(n-1)}\right) .
$$

Let us assume now that $a-\sigma^{(n)} \geq b$ holds for all $n$; this is obviously a practical assumption. Then

$$
\left|\sigma^{(n+1)}-\sigma^{(n)}\right| \leq \frac{B \nabla_{j+1} t}{2 b^{2}}\left|\sigma^{(n)}-\sigma^{(n-1)}\right|,
$$

whence

$$
\left|\sigma^{(n+1)}-\sigma^{(n)}\right| \leq\left(\frac{B \nabla_{i+1} t}{2 b^{2}}\right)^{n}\left|\sigma^{(1)}-\sigma^{(0)}\right|=\left(\frac{B \nabla_{i+1} t}{2 b^{2}}\right)^{n}\left|\nabla_{i+1} t \Sigma_{0}\right| .
$$

The sequence $\sigma^{(n)}$ is thus convergent provided $\left|B \nabla_{i+1} t / 2 b^{2}\right|<1$, a condition which is found to be satisfied in practice. Thus, for example, for the values $a=1 \mathrm{inch}, b=$ $(1 / 3)$ inch, $\nabla_{1}=1 / 100, u_{m}=5000, \rho=150, c=1 / 2, k=1 / 3000, F=3000, \nabla_{i+1} t=$ $1 / 4$, and $U_{1, j+1}=4000$ there results $B \nabla t / 2 b^{2} \approx 1 / 100$, so that the iteration will converge rapidly. If we denote $\lim _{n \rightarrow \infty} \sigma_{i}^{(n)}$ by $\sigma_{i}$, then it is seen that

$$
\left|\sigma_{i}-s_{i}\right| \leq \text { Const } \nabla_{i} t \text {, for } 0 \leq j \leq j_{\max } \text {. }
$$

V. System of difference equations. The development of the difference equation analogous to the differential equation (17) for interior points is somewhat more laborious. Applying Eq. (17) at the point $\xi_{i}, t_{i+1}$, replacing $\partial^{2} U / \partial \xi^{2}$ by Eq. (16), $\partial U / \partial t$ by Eq. (13), and $\partial U / \partial \xi$ by the central divided difference approximation obtained from Eqs. (12) and (15),

$$
\left(\frac{\partial U}{\partial \xi}\right)_{i, j+1}=\frac{1}{2}\left[\frac{U_{i+1, i+1}-U_{i, j+1}}{\nabla_{i+1}}+\frac{U_{i, j+1}-U_{i-1, j+1}}{\nabla_{i}}\right]+C_{4}|\nabla \xi|,
$$

there is obtained, after some calculation, with $p_{i}=\nabla_{i+1} / \nabla_{i}$,

$$
\begin{aligned}
& -\left[2 r_{i i} \frac{p_{i}^{2}}{1+p_{i}}-\frac{1-\xi_{i}}{2\left(a-s_{j}\right) \nabla_{i}} s_{i}^{j} \nabla_{i+1} t\right] U_{i-1, i+1} \\
+ & {\left[1+2 p_{i} r_{i j}-\left(1-p_{i}^{-1}\right) \frac{1-\xi_{i}}{2\left(a-s_{i}\right) \nabla_{i}} s_{i} \nabla_{i+1} t\right] U_{i, j+1} } \\
& -\left[2 r_{i i} \frac{p_{i}}{1+p_{i}}+\frac{1-\xi_{i}}{2\left(a-s_{i}\right) \nabla_{i}} p_{i}^{-1} s_{i} \nabla_{i+1} t\right] U_{i+1, i+1} \\
= & U_{i j}+\frac{r_{i j}}{4}\left(\frac{k^{\prime}}{k}\right)_{i j}\left[U_{i+1, i}-\left(1-p_{i}\right) U_{i i}-p_{i} U_{i-1, i}\right]^{2} \\
+ & \nabla_{t}\left[C_{5}|\nabla \xi|+C_{6}\left(\nabla_{t}\right)\right] .
\end{aligned}
$$

$\dagger$ For convenience in writing the subscript $j+1$ has been deleted in some of the following equations. 
Let us, finally, turn to the condition (9) expressing the insulation of the rear boundary. The difference equation representing this condition may be obtained either directly by means of Eqs. (12), (15), (13), and (17), or by applying Eq. (24) to the case $i=n$. In this case, since $\xi_{n}=1$, and assuming $U_{n+1, i}=U_{n-1, i}, \nabla_{n+1}=\nabla_{n}$, there is obtained immediately:

$$
-2 r_{n j} U_{n-1, i+1}+\left(1+2 r_{n i}\right) U_{n, j+1}=U_{n i}+\nabla t\left[C_{7}|\nabla \xi|+C_{8}(\nabla t)\right] .
$$

It follows, then, that the system of differential equations (1), (2), (3) may be approximated by the following system of difference equations:

$$
\begin{gathered}
\left(1+2 r_{0 i}\right) T_{0, i+1}-2 r_{0 i} T_{1, i+1}=T_{0 i}+2 r_{0 i}\left(a-s_{j}\right) \nabla_{1} k_{0 i}^{-1} q_{i+1} \quad \text { if } \quad T_{0, i+1}<u_{m} \\
\Sigma_{i+1}^{(n)}=A_{i+1}-B_{i+1}\left(a-\sigma_{i+1}^{(n)}\right)^{-1}, \\
\sigma_{i+1}^{(n+1)}=\sigma_{i}+\left(\nabla_{i+1} t / 2\right)\left[\Sigma_{i}+\Sigma_{i+1}^{(n)}\right], \quad n=0,1,2, \cdots \\
T_{0, i+1}=u_{m}, \\
-\left[2 r_{i i} \frac{p_{i}^{2}}{1+p_{i}}-\frac{1-\xi_{i}}{2\left(a-\sigma_{j}\right) \nabla_{i}} \Sigma_{i} \nabla_{i+1} t\right] T_{i-1, i+1}+ \\
+\left[1+2 r_{i j} p_{i}-\left(1-p_{i}^{-1}\right) \frac{1-\xi_{i}}{2\left(a-\sigma_{j}\right) \nabla_{i}} \Sigma_{i} \nabla_{i+1} t\right] T_{i, i+1} \\
-\left[2 r_{i i} \frac{p_{i}}{1+p_{i}}+\frac{1-\xi_{i}}{2\left(a-\sigma_{i}\right) \nabla_{i}} p_{i}^{-1} \Sigma_{j} \nabla_{i+1} t\right] T_{i+1, i+1} \\
=T_{i j}+\frac{r_{i i}}{4}\left(\frac{k^{\prime}}{k}\right)_{i i}\left[T_{i+1, i}-\left(1-p_{i}\right) T_{i j}-p_{i} T_{i-1, i}\right]^{2}
\end{gathered}
$$

Equation (30) applies to $i=1,2, \cdots, n-1$.

Equations (26), (29) and (30) revert to the corresponding Eqs. (10), (12), and (8) of [1] in case of non-melting; it is necessary only to replace $a \nabla_{i}$ by $\Delta x, \nabla_{i} t$ by $\Delta t, \sigma_{i}$ and $\sum_{i}$ by 0 .

For equal values of $\nabla x$ and $\nabla t$ the equations become essentially identical with those of [3], with the exception of the relationship (19) and (20) for the rate of melting.

VI. Integration procedure. The onset and stopping of melting presents some minor problems in the solution of the system (26) through (30) of difference equations. A possible approach is the following:

(a) Initially, at $j=0, \sigma_{0}=0, \sum_{0}=0$. Eqs. (26), (29), and (30) represent $n+1$ linear equations in the $n+1$ unknowns $T_{i i}$, for $i=0,1, \cdots, n$. The matrix of the linear system is tri-diagonal, and may thus be treated by the method of solution of [1].

This step is repeated, for an arbitrary choice of time increments $\nabla_{1+1} t$, so long as $T_{0, i+1}<u_{m}$.

(b) Eventually it may happen that $T_{0 i}=T_{0}\left(t_{j}\right)<u_{m}$ but $T_{0, j+1}=T_{0}\left(t_{j}+\nabla_{i+1} t\right)>$ $u_{m}$. Then the solution of Eqs. (26), (29), and (30) is carried out for a progressively reduced sequence $\nabla_{i+1} t$, until $T_{0, j+1}=T_{0}\left(t_{j+1}\right)$ satisfies $\left|T_{0}\left(t_{j+1}\right)-u_{m}\right| \leq \epsilon, \epsilon>0$ prescribed. The values of $T_{i, i+1}$ for $i=1,2, \cdots, n$ are now assumed to be correct.

Placing in Eq. (27) $n=0, \sigma_{i+1}^{(0)}=\sigma_{i}, \sum_{j+1}^{(0)}=\sum_{i}, \nabla_{i+1} t=t_{i+1}-t_{i}$, and $\left(T_{\xi}\right)_{0, i+1}$ evaluated from $T_{0, i+1}=u_{m}, T_{1, i+1}, T_{2, i+1}, T_{3, i+1}$, by Eq. (12), there is obtained the 
value of $\sum_{j+1}^{(1)}$, and then $\sigma_{i+1}^{(1)}$ by Eq. (28a). This operation is repeated until convergence to limiting values $\sigma_{i+1}>0$ has been achieved.

(c) The integration is now resumed, for the equations (28b), (29), and (30), where $\sigma_{i}, \sum_{i}$ are replaced, respectively, by the values of $\sigma_{i+1}$ and $\sum_{i+1}$ obtained by (28a), (27). While $\nabla_{i+1} t$ is arbitrary, it should be restricted by the requirement that $\nabla_{i+1} s<$ $\nabla_{1} x$ or $\sum_{i} \nabla_{i+1} t<\left(a-\sigma_{i}\right) \nabla_{1}$. Then Eq. (27) is utilized again.

This step is repeated so long as $\sum_{i+1}>0$.

(d) Eventually it may happen that $\sigma_{i+1}<0$. Equations (28b), (29), (30) are now solved with a sufficiently reduced value of $\nabla_{i+1} t$ so as to produce $\sum_{i+1}=0$, to within a prescribed tolerance. The next step then proceeds as in (a).

VII. Applications. In testing the method described above one may conveniently use the case of the semi-infinite slab of a material which possesses constant thermal properties $k, c, \rho$. If it is further assumed that the heat input $q$, and the initial temperature distribution $f(x)=u_{0}$ are constant, then the solution, before melting starts, of the problem is given by [4]

$$
u(x, t)-u_{0}=\frac{2 q}{k \pi^{\frac{1}{2}}}\left[(\alpha t)^{\frac{1}{2}} \exp \left(\frac{-x^{2}}{4 \alpha t}\right)-\frac{x}{2(\alpha t)^{\frac{1}{2}}} \int_{x}^{\infty} e^{-\sigma^{2}} d \sigma\right] .
$$

For the time $t_{m}$ at which melting starts, since $u\left(0, t_{m}\right)=u_{m}$, there is obtained

$$
t_{m}=\pi k c \rho\left[\left(u_{m}-u_{0}\right) / 2 q\right]^{2} .
$$

Further, as Landau [2] pointed out, there exists a steady state solution of the system of differential equations (1), (2), (3) for the case of the semi-infinite slab; as $t \rightarrow \infty$

$$
\begin{gathered}
s^{\cdot} \rightarrow s_{\infty}^{\cdot}=q\left\{\left[\rho F+c\left(u_{m}-u_{0}\right)\right]\right\}^{-1}, \\
s(t) \rightarrow s_{\infty}=s_{\infty} t-k q^{-1}\left(u_{m}-u_{0}\right)
\end{gathered}
$$

and, in general,

$$
s^{\cdot}<s_{\infty}^{\cdot}, \quad s_{\infty}<s(t) \leq s_{\infty}^{\cdot}\left(t-t_{m}\right) .
$$

The results obtained by means of a program for the IBM 704 machine based on the system of difference equations discussed here have been quite satisfactory. In particular, Eq. (31), indicating the onset of melting, and Eq. (32) for the rate of melting, have been found to hold to high degrees of accuracy.

\section{REFERENCES}

1. M. Lotkin, The numerical integration of heat conduction equations, J. Math. Phys. 37, 178-187 (1958)

2. H. G. Landau, Heat conduction in a melting solid, Quart. Appl. Math. 8, 81-94 (1950)

3. J. D. Brown and R. E. Mascola, Numerical solution of the heat conduction equation.....," AVCO RAD-2-TM-58-011, 1958

4. R. V. Churchill, Modern operational mathematics in engineering, McGraw-Hill, New York, 1944, p. 108 